\title{
The Use of Solvent-Gel Systems for the Cleaning of PMMA
}

\author{
Stefani Kavda ${ }^{1}$, Emma Richardson ${ }^{2}$ and Stavroula Golfomitsou ${ }^{1}$ \\ ${ }^{1}$ UCL Qatar, University College London, Doha, Qatar \\ ${ }^{2}$ Department of History of Art, University College London, London, UK
}

\begin{abstract}
This paper discusses the use of solvent-gel systems for the cleaning of poly (methyl methacrylate) (PMMA) surfaces. Aqueous polymer gel systems have been introduced to the conservation field with a particular focus on paintings, painted surfaces of wooden artefacts and stone. However, their application on plastics is very recent. Statistically designed experiments aimed to assess the efficiency and damage potential of materials constituting selected solvent-gel cleaning systems. The effect of the free solvents (deionized water, ethanol, isopropanol and petroleum ether), the hydrogel carriers (Agar, Pemulen ${ }^{\mathrm{TM}} \mathrm{TR}-2$, Carbopol ${ }^{\circledR} \mathrm{EZ}$ 2-Ethomeen ${ }^{\circledR} \mathrm{C}$ 25 and $80 \%$ hydrolysed PVAc-borax) and their combinations after 5 and 60 minutes of application time on PMMA surfaces, were evaluated through qualitative visual microscopic observation and SEM imaging, and quantitative weight change measurements. Visual observations revealed that the action of solvents (i.e. ethanol and isopropanol) was moderated by gellation (i.e. Agar), while other gel systems (i.e. Pemulen) were unsatisfactory. Results of gravimetric studies showed that most solvent-gels resulted in inconsistencies and large weight changes.
\end{abstract}

\section{INTRODUCTION}

Plastic objects that were once mass produced or uniquely created by celebrated artists, have now acquired a collectible value due to their scarcity, unconventional design [1] or broad influence as iconic works of art. Their artistic value depends on their condition, severely reliant on their conservation. The aesthetic quality of artworks made of poly (methyl methacrylate) (PMMA) - one of the most frequently used plastics in art and design [2,3] - is of immense importance. Their ability to transmit light is integral to their character. Being thermoplastic, PMMA also has the capacity to be softened with temperature, making it easy to be molded into various shapes. For all these reasons PMMA has been cherished by artists, such as Craig Kaufmann, Yoko Ono, John Chamberlain, Donald Judd and design companies like Kartell and Guzzini. Helen Pashgian (2010) [4] of the 'Light and Space' movement, established in California during the sixties, stated that "On any of these works, if there is a scratch ... that's all you see". Pristine transparent surfaces are hard to clean, mainly because surface modifications can be easily visible [5], and also call for a very low tolerance to physical damage [6] making these objects challenging to care for [7]. There is a general lack of a systematic approach regarding the cleaning of plastics or an evaluation of all possible risks. For example, removing accumulated surface dirt might result in loss of transparency and other aesthetic qualities. In this preliminary 
study we present work intending to assess the damage potential of the materials employed in gel cleaning methods and identify the factors that influence their use on PMMA. Experimental work initially examined hydrogel matrices and solvents alone, in order to understand their behaviour and interaction with PMMA. Solvent-gels were also assessed.

PMMA because of its amorphous nature is vulnerable to solvents which might instigate cracking $[8,9]$ and demands a more controlled solvent action. Gels could offer this, through their assumed potential to limit free solvent penetration into cracks and pores of the surface, while minimizing the risk of dissolution of the material $[10,11]$. As the use of gels on plastic surfaces has not been studied in depth to date, it is imperative to adopt a systematic approach to evaluate their effect on pristine acrylic surfaces, without dirt, to use as a reference point for further studies.

\section{EXPERIMENTAL DETAILS}

General full-factorial experiments were designed in Minitab® to examine the effect and interactions of hydrogels, free solvents, and application time (Table I). Treatments were employed on new transparent/colourless, mechanically cut PMMA samples ( 25 x 25 x $0.38 \mathrm{~mm}$ ), obtained by Goodfellow UK (ME303001), for $5 \mathrm{~min}$ and $60 \mathrm{~min}$, in order to collect reference data about the material in its original condition without the implications of soiling and degradation. Applications were carried out in triplicate for hydrogels and free solvents, and duplicate for solvent-gels, and were randomized to prevent systematic error.

Table I. Variables (Factors and levels) in the design of the cleaning experiments.

\begin{tabular}{|c|c|c|c|}
\hline Factor & 1. Gels & 2. Solvents & 3. Application time \\
\hline \multirow[t]{4}{*}{ Levels } & 1. Agar Agar & 1. Water (DI water) & $1.5 \mathrm{~min}$ \\
\hline & 2. Carbopol®-Ethomeen ${ }^{\circledR}$ & 2. Ethanol $(\mathrm{EtOH})$ & 2. $60 \mathrm{~min}$ \\
\hline & 3. Pemulen ${ }^{\mathrm{TM}}$ TR2 & 3. Isopropanol (IPA) & \\
\hline & 4. PVAc-Borax & 4. Petroleum ether (PET) & \\
\hline
\end{tabular}

Hydrogels were prepared as aqueous systems, as water is necessary for the gelling process, whereas all solvent-gels were loaded with $20 / 80 \mathrm{wt}$. $\%$ solvent/DI water mixtures (see 2. Solvents in Table I). Agar (Sigma-Aldrich®) was dissolved in DI, boiling water to form a $2 \mathrm{wt}$.\% solution $(\mathrm{pH}$ 7.0). Once left to cool, the solution was re-heated to improve the water retention properties of the gel [12]. The same procedure was repeated for the solvent-gels. Carbopol@ EZ2Ethomeen ${ }^{\circledR}$ C-25 polymeric dispersions (Figure 1) were prepared after a recipe suggested by Stavroudis and Blank [13], Pemulen ${ }^{\mathrm{TM}}$ TR-2 gels (1 wt.\% Pemulen/5 wt.\% triethanolamine 
[TEA]) after a recipe by Stavroudis [14], and 80\% hydrolysed poly (vinyl acetate) (PVAc)/borax (5 wt.\%/1 wt.\%) according to Angelova et al. [15] (Figure 1).
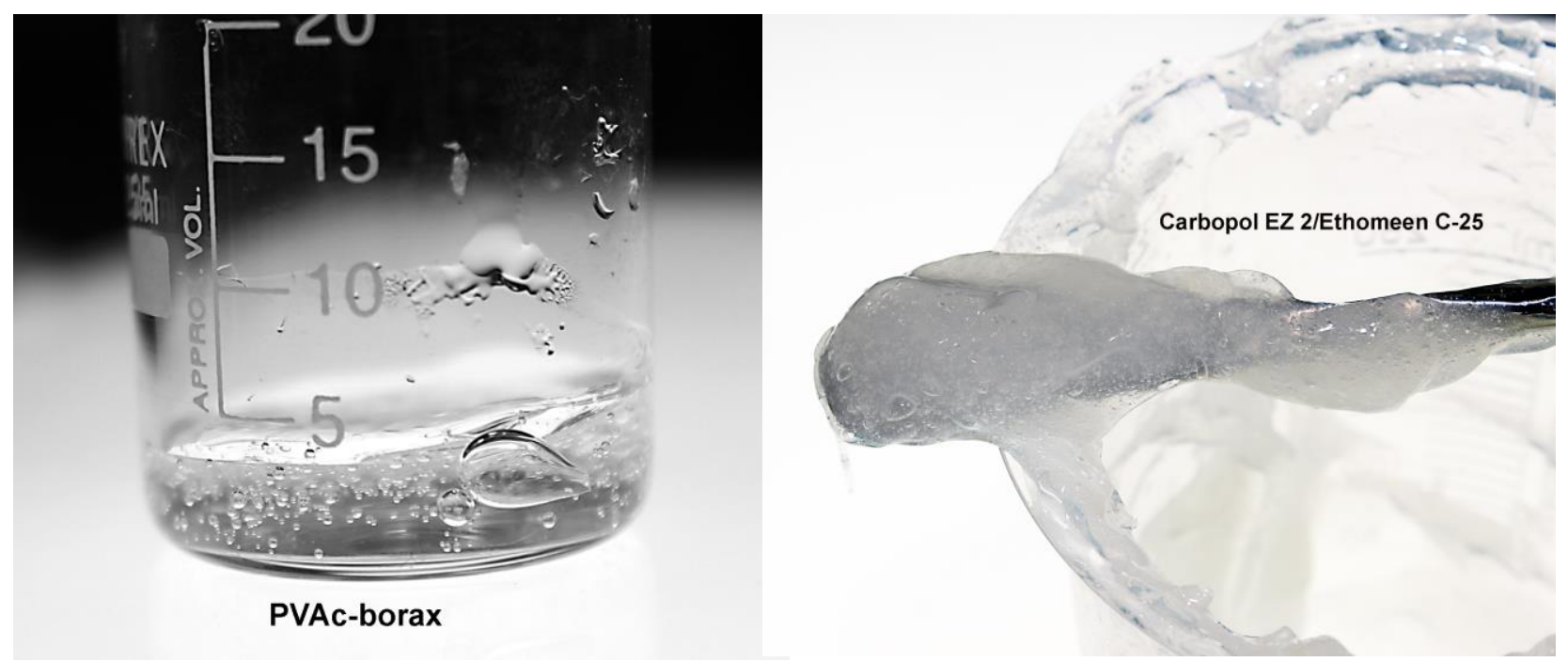

Figure 1. Freshly prepared PVAc-borax and Carbopol-Ethomeen. Images by Stefani Kavda.

Application of gels was carried out with a Teflon spatula. Agar gels were placed on sample surfaces with a glass weight on top, to improve contact. Carbopol-Ethomeen and Pemulen, being paste-like were applied with the spatula, while PVAc-borax was first flattened between two glass slides and then placed on the samples. All gels were prepared or applied as $2 \mathrm{~mm}$ thick. Gels were removed by lifting or wiping them with Teflon spatula, followed by three separate cotton swabs moistened with DI water, linearly rolled towards one direction.

Leica M205 A3D stereomicroscope under raking light replaced the traditional digital photography to eliminate the scattering effect of light and capture the transparent and highly polished surface of PMMA. For a more detailed image of the surface micro topography, reference and treated samples were gold-coated and observed using a JEOL JSM-6610LV SEM in high vacuum mode at $10 \mathrm{kV}$. Gravimetric analysis was carried out before and after treatment to provide quantitative evidence of changes in the material. Treated samples were left at ambient temperature for 24 hours prior to weighing with Sartorius MSE225S-000-DU Cubis Semi-Micro Balance (accuracy of $0.01 \mathrm{mg}$ ).

\section{RESULTS}

\section{Ease of gel handling}

PVAc-borax and Agar were easy to prepare. The former was challenging to apply, due to being self-healing, but easy to peel. Agar was not compatible with PET, but introduction of the solvent didn't prevent gellification. Agar gels were rigid, thus easily placed and removed in one piece. The texture of Carbopol and Pemulen was quite tacky, resembling a paste easy to apply, 
but hard to remove leaving greasy residues. Dissociation of the gels and residues will be further explored.

\section{Hydrogels}

Agar hydrogels caused small weight variations to the samples, and insignificant abrasions visible only under the SEM. Samples treated with Carbopol-Ethomeen hydrogels exhibited an irregular behaviour, indicating the uncontrollable action of the gel, especially with increased application time. PVAc-borax hydrogels induced weight gain, with smaller fluctuations after 60 min, suggesting that gels behaved better with increased time. Visually, surfaces were left minimally blemished. Samples treated with Pemulen hydrogels displayed fluctuating weight changes, and their surface condition was inferior to samples treated with the other hydrogels, appearing greasy and blemished (Figure 2A). Pemulen left a large amount of residues, contrary to the other hydrogels, which left negligible or no residues. Based on the statistical analysis of weight changes, hydrogels did not affect the outcome of treatments, and were thus considered insignificant at this stage, where experiments were run on new, unsoiled surfaces.

\section{Free solvents}

There was no clear pattern of weight change with free DI water and PET, with group replicates exhibiting behaviour of both loss and gain of weight, however causing bigger changes after longer time exposures, possibly due to their low volatility. The opposite holds true for $\mathrm{EtOH}$ and IPA, for which prolonged time resulted in a reduced weight loss, due to greater and more rapid solvent evaporation. Visually, PET left surfaces minimally blemished, while EtOH and IPA caused non-uniform formation of dull areas (Figure 2C). Under the SEM, this was identified as chemical surface alteration resulting from contact with the solvent (Figure 2E). $\mathrm{EtOH}$ was identified as the solvent having the greatest effect on the treatments, followed by IPA and DI water.

\section{Solvent gels}

The weight loss of samples induced by the free solvents, has been intensified with the presence of Agar (Figure 3). This suggested that the gel possibly encouraged the loss of residual unreacted methyl methacrylate (MMA) monomer left from incomplete free radical polymerisation reactions, or low-molecular-weight fragments from initial degradation reactions (bond cleavage), to evaporation or/and extraction into the gel matrix. The nature of the polymer components is unknown at this time, but results of Py-GC/MS will soon become available. In relation to weight fluctuations, EtOH and DI water in Agar showed a slight improvement with time, compared to their free standing action, possibly indicating that the gel controlled the solvent action. This is supported by visual analysis showing a slight improvement in surface condition; the extent of non-uniform dull areas seen after free EtOH and IPA has been reduced with introduction of the solvents in Agar. Visual analysis of Agar/PET did not exhibit distinct differences from the free standing action of the solvent. IPA and PET/Agar gels did not offer encouraging results. Very few gel deposits were detected by SEM after solvent-based Agar gel treatment.

The unpredictable weight fluctuations after Carbopol-Ethomeen hydrogels, appeared to have become more consistent with the addition of solvents. EtOH/Carbopol caused the weight increase of samples, the opposite behaviour of samples treated with free-standing EtOH (Figure 3). This indicated that the gel limited solvent evaporation, resulting in an increased contact time 
between solvent and PMMA, and consequently in potentially aggravating PMMA dissolution. Visually, EtOH/Carbopol appeared to be too aggressive on PMMA (Figure 2B). This observation was confirmed by detection of scratches, and non-uniform opaque areas, also encountered after treatment with free EtOH. After the 60-minute application, SEM revealed significant Carbopol residues, rendering all solvent-Carbopol treated surfaces appear dull. Although from a weight change perspective, Carbopol/IPA appeared least invasive, surfaces were left severely abraded.

Solvent-Pemulen treated samples showed a similar pattern of weight change with samples treated with solvent-Carbopol gels; Pemulen with EtOH and PET induced great mass increases (Figure 3), by possibly limiting solvent evaporation. Pemulen/PET caused the maximum weight gain, rendering the gel system unsuitable for use on PMMA. Pemulen/DI water and Pemulen/IPA caused the inconsistent weight behaviour of samples. Surfaces after solvent/Pemulen application, visually appeared as greasy as samples after Pemulen hydrogels. Pemulen with EtOH and IPA intensified this effect and induced heavy scratching. A large amount of residues was detected by SEM (Figure 2D).

Samples after solvent/PVAc gels, especially when coupled with EtOH, exhibited the least alterations in weight compared to the other solvent/gels (Figure 3). The presence of PVAc possibly resulted in more solvent control than other gel systems, but still caused more weight changes than treatment with free solvents. Visually, solvent/PVAc induced surface alterations, such as abrasions and blemishes, and occasionally, chemical surface alteration from contact with the solvents, exhibited as loss of transparency under the SEM. There were no residues nor abrasions apparent after any of the solvent-PVAc systems, phenomenon attributed to their limited adhesion to the polymer surface [15]. No swelling was visible in any of the hereby discussed solvent-gel treated samples.

It was evident from the statistical analysis of the weight changes of samples treated with solvent-gels, that the gel type played the most crucial role; it affected the most the treatment outcome, and the PMMA surface, especially in samples treated with Agar or Pemulen. In their majority, interactions between gels and solvents also strongly affected the treatments and their effect on PMMA. Results showed that Agar affected the treatment the most when coupled with IPA and PET, Pemulen with EtOH and PET, Carbopol with EtOH, and PVAc with PET. Interestingly, application time generally didn't affect the treatments, except in cases of prolonged PMMA exposure to systems with either Carbopol or EtOH. At this stage, interpretation of whether the process is affected in a positive or a negative way, is not possible. 


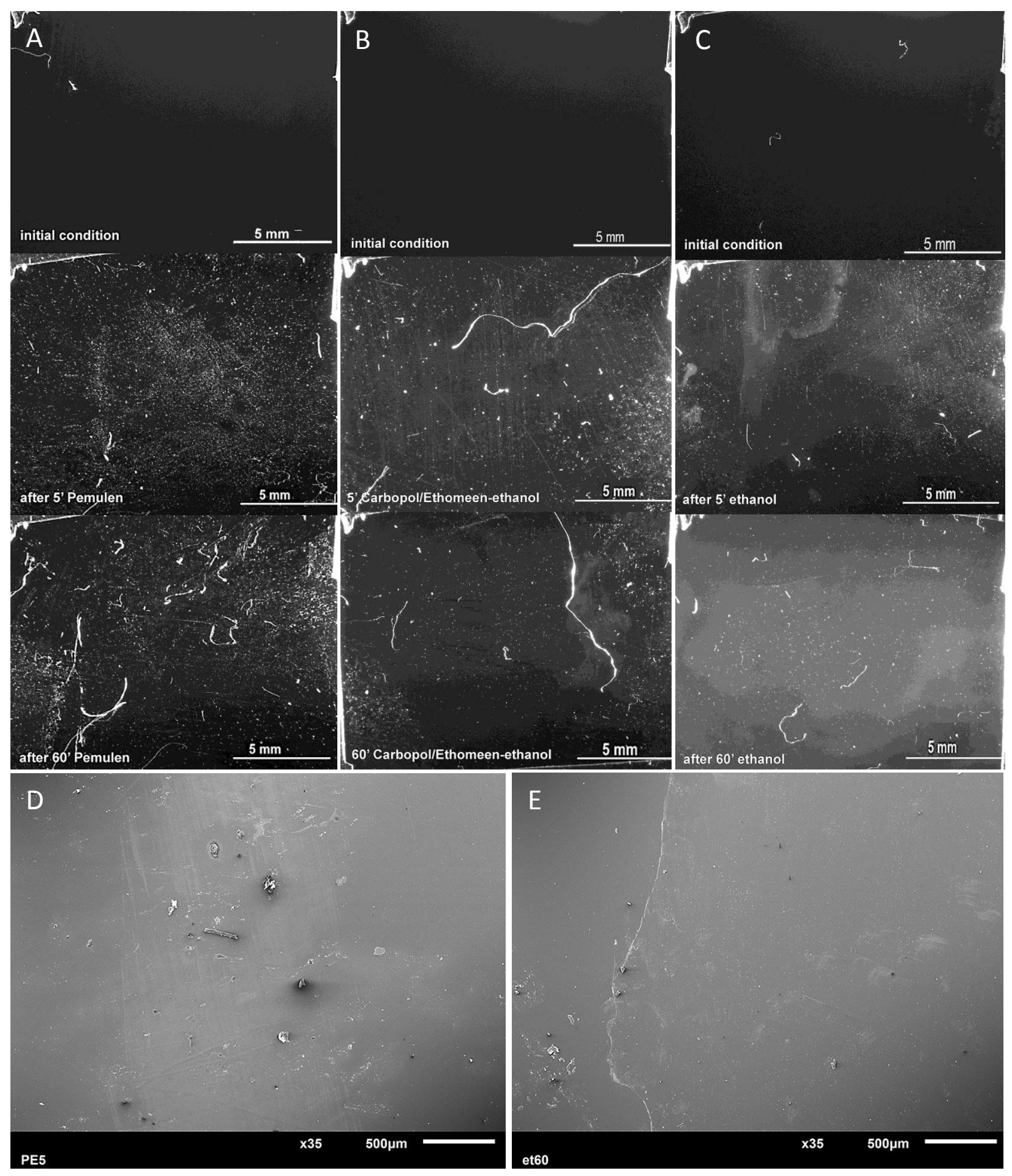

Figure 2. A-C: Stereomicroscopic images of PMMA samples under raking light (x7.81) at three phases, before treatment and after 5, and 60 minutes of application: A. Surfaces after Pemulen hydrogels appeared greasy, blemished and with large amounts of gel residues. B. EtOH/Carbopol caused scratches after $5 \mathrm{~min}$ and non-uniform opaque areas after $60 \mathrm{~min}$. C. Free EtOH caused non-uniform formation of dull areas. D-E: SEM micrographs (x35): D. Blemishes and large amounts of residues after Pemulen gels. E. Surface alteration due to exposure to EtOH. 


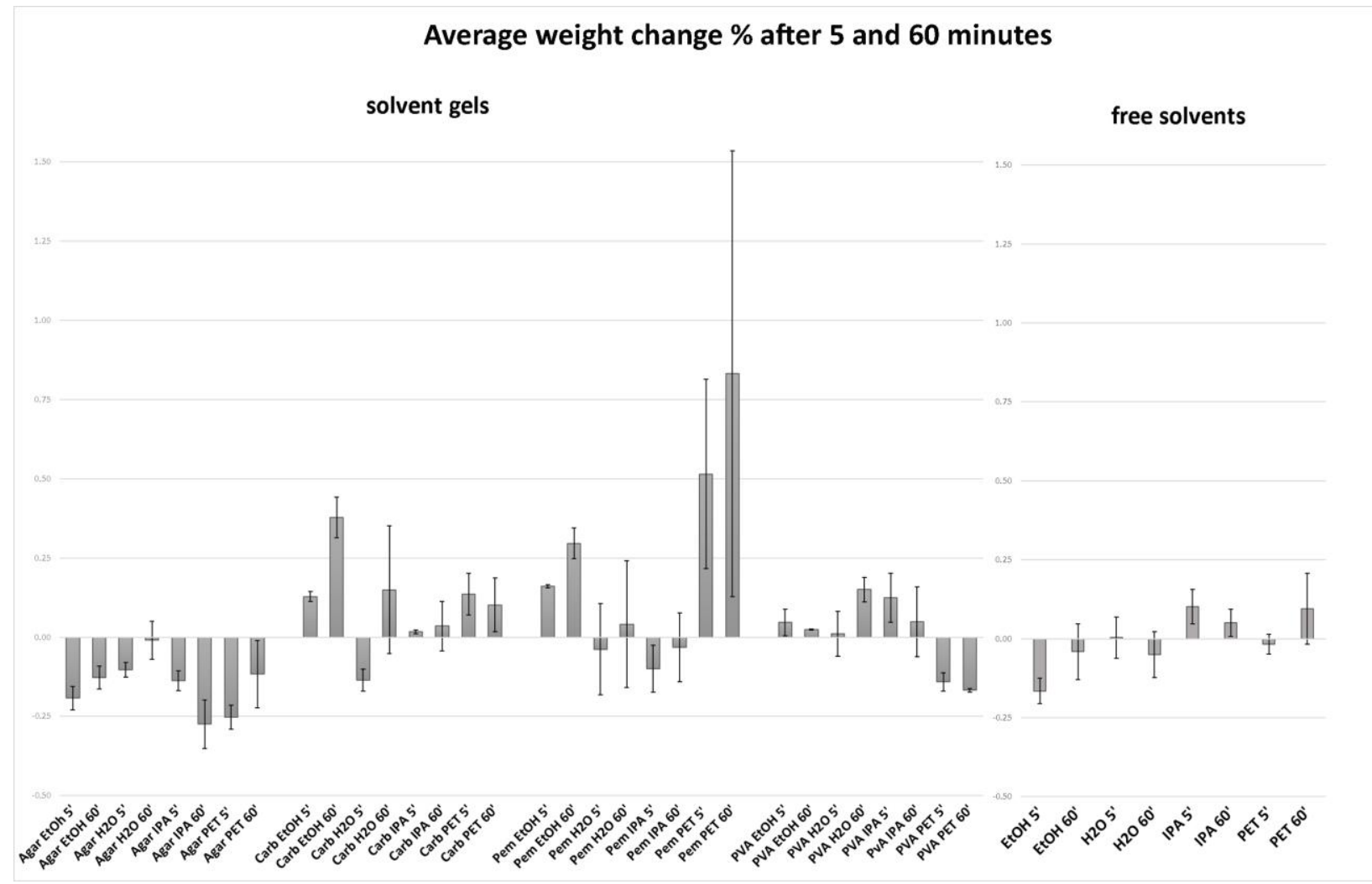

Figure 3. Average weight change \% of PMMA samples after solvent-gels and free solvents for 5 and $60 \mathrm{~min}$.

\section{DISCUSSION}

The use of gels has undoubtedly influenced the performance of solvents, however, the exact mechanisms of action, on PMMA, need further study. The most common degradation mechanisms of PMMA are crosslinking and bond cleavage, which result in conflicting phenomena: the former increases the molecular weight, whereas the latter causes its reduction [16]. Interestingly, these phenomena often occur simultaneously [17], and can either take place to the main-chain or the side groups of polymer molecules [18]. Solvents too, are capable of decreasing the molecular weight of a polymer, by extracting components, or dissolving it [5]. It is hoped that these processes will be better understood in light of the FTIR analysis currently undertaken.

In cases of increased weight losses, the gel system was considered too damaging for use on PMMA. This can be attributed to polymer exposure to the solvent, which would lead to the local dissolution and extraction of volatile PMMA components, such as methyl formate, methane, hydrogen and carbon oxides [19, 20]. It is assumed that gels offer some control over solvent action, by reducing its evaporation rate and increasing contact between the carried solvent and the treated surface. Samples with large weight changes in this study, also tended to exhibit adverse surface effects, such as scratching and loss of transparency. However, follow-up experiments will be carried out to examine whether the increased solvent exposure of PMMA 
surfaces, resulting from the gel control over the solvent action, can be an advantage when dealing with surfaces bearing accumulated dirt.

Changes in the optical properties of the PMMA surfaces, as well as weight variations, need to be further investigated in order to agree on the acceptable level for use in conservation of transparent acrylics. The results showed inconsistencies, which did not allow clear interpretation and drawing of definite conclusions. The use of triplicates and duplicates was not sufficient for this type of experimental work; thus additional experiments will be carried out on increased number of replicates to evaluate the experimental outcomes and errors.

\section{CONCLUSIONS}

The use of statistical methods for the organisation and the interpretation of data was necessary, especially compared with the observations of weight changes and visual microscopic assessment, to draw conclusions in an objective manner. This study was fundamental in laying out the net effect of the materials comprised in solvent-gels, on new, pristine, transparent and colourless PMMA surfaces. By documenting their interaction with the polymer surface, and damage potential, we can then proceed to studying their combined effect on PMMA surfaces bearing accumulated dirt.

Experimental results showed that PET behaved better free-standing than gellated. IPA/Agar and PET/Agar did not offer encouraging results. Samples treated with Carbopol-Ethomeen gels presented irregularities and dispersive weight values, and visually were unsatisfactory. The condition of samples after solvent-Pemulen gels appeared inferior, especially due to greasy residues. PVAc-borax gels, especially with $\mathrm{EtOH}$, exhibited the most controlled action, reducing scratching and gel residues. Visually, EtOH and IPA caused the most surface damage, often moderated by gellation. In terms of visual damage, there was an inclination among solvent/gels in this study to perform slightly better after longer application times, even though statistical analysis of the weight changes evaluated that application time was a statistically insignificant factor.

Having identified factors of greatest significance, experimental errors and biases, follow-up cleaning experiments will be carried out. These will include more factors, such as surface condition and dirt type. It is clear that additional work with a greater number of replicates is essential to showcase the general trend, before a complete understanding of the pattern of behaviour of solvent-based gels.

\section{ACKNOWLEDGMENTS}

Stefani Kavda would like to thank Qatar Foundation for funding the Ph.D. research, and acknowledge the MRS TC3 session organizers and XGLab S.R.L. for the partial support to attend the conference. Authors would like to thank Dr. Lora Angelova for her valuable collaboration and advice, Professor Thilo Rehren for his continuous support, and Philip Connolly for his help with the SEM imaging. We are also grateful to Stephanie Black for technical assistance. 


\section{REFERENCES}

1. M. Pugliese, and F. Waentig, in Preservation of Plastic Artefacts in Museum Collections, edited by B. Lavédrine, A. Fournier, and G. Martin (Editions du Comité des travaux historiques et scientifiques, Paris, 2012), p. 23.

2. D. Sale, in An evaluation of eleven adhesives for repairing poly (methyl methacrylate) objects and sculpture, edited by D. Grattan, (Saving the twentieth century: the conservation of modern materials: proceedings of a conference Symposium 91: Saving the Twentieth Century, Ottawa, Canada, 1993) pp. 325-340.

3. F. Waentig, Plastics in Art, (Michael Imhof Verlag, Petersberg, 2008) p. 272.

4. M. Simms, CAA Reviews, (2015).

5. Y. Shashoua, in Preservation of Plastic Artefacts in Museum Collections, edited by B. Lavédrine, A. Fournier, and G. Martin (Editions du Comité des travaux historiques et scientifiques, Paris, 2012), pp. 219-223.

6. L. Dei, in Nanoscience for the Conservation of Works of Art, edited by P. Baglioni, and D. Chelazzi (The Royal Society of Chemistry, Cambridge, 2013), p. 7.

7. R. Rivenc, E. Richardson, and T. Learner in The LA Look from Start to Finish: Materials, Processes and Conservation of Works by the Finish Fetish Artists, edited by J. Bridgland, (ICOM-CC 16th triennial conference 19-23 September 2011: preprints, Lisbon, Portugal, 2011) p. 6.

8. S. Akhurst, in Plastics: looking at the future and learning from the past: papers from the conference held at the Victoria and Albert Museum, London: 23-25 May 2007, edited by B. Keneghan, and L. Egan (Archetype Publications Ltd, London, 2008), p. 160-162.

9. Y. Shashoua, Conservation of Plastics: materials science, degradation and preservation, (Butterworth-Heinemann, Oxford, 2008).

10. E. Carretti, M. Bonini, L. Dei, B.H. Berrie, L.V. Angelova, P. Baglioni and R.G. Weiss, Acc. Chem. Res. 43 (6), 751-760 (2010).

11. R. Wolbers, Cleaning Painted Surfaces: Aqueous Methods, (Archetype Publications, London, 2000).

12. P. Cremonesi in Rigid Gels and Enzyme Cleaning, edited by M.F. Mecklenburg, A.E. Charola, and R.J. Koestler, (New insights into the cleaning of paintings. Proceedings from the Cleaning 2010 International Conference, Washington, DC, 2013) pp. 179-183.

13. C. Stavroudis, and S. Blank, WAAC Newsletter 11 (2), 2-10 (1989).

14. C. Stavroudis, WAAC Newsletter 34 (2), 19 (2012).

15. L.V. Angelova, B.A. Ormsby, and E. Richardson, Microchem. J. 124, 311-320 (2016)

16. D. M. Wiles, in Changes in polymeric materials with time, edited by D. Grattan, (Saving the twentieth century: the conservation of modern materials: proceedings of a conference Symposium 91: Saving the Twentieth Century, Ottawa, Canada, 1993) pp. 105-112.

17. G. Wypych, Handbook of Material Weathering, (ChemTec Publishing, Toronto, 2013) p. 330.

18. T. Lister, and J. Renshaw, Conservation Chemistry- an introduction, (Royal Society of Chemistry, London, 2004) p. 19.

19. J. O. Choi, J. A. Moore, J. C. Corelli., J. P. Silverman and H. Bakhru, J. Vac. Sci. Technol. 6 (6), 2286-2289 (1988).

20. T. Çaykara and O. Güven, Polym. Degrad. Stab. 65 (2), 225-229 (1999). 